\title{
Driver Modeling and Implementation of a Fuel-saving ADAS
}

\author{
Invited Session Paper: SMC2018:H22 \\ James M. Fleming, Xingda Yan, Craig K. Allison, Neville A. Stanton, Roberto Lot \\ Faculty of Engineering and the Environment, \\ University of Southampton, UK
}

\begin{abstract}
Controlling vehicle velocity, by coaching the driver to eco-drive with an advanced driver assistance system (ADAS), is a promising method to decrease fuel consumption and greenhouse gas emissions for combustion engine-driven road vehicles. By using optimal control techniques, such a system may find velocity profiles in real-time that minimize fuel consumption. This is particularly useful to recommend the optimal time to initiate coasting, which is otherwise difficult to estimate by a driver. However, this ADAS should not choose velocities and accelerations that the driver will dislike, such as those that leave too much or too little space to the preceding vehicle, or those that take corners at high speed. To remedy this, we introduce an optimal control model of acceleration that mimics drivers' behavior and combine this with a model of fuel consumption to trade-off driver preferences and fuel savings. We give examples of the velocity profiles recommended in a typical driving scenario to demonstrate the potential fuel savings. Finally, we give details of a prototype system, which has recently been implemented in the driving simulator at the University of Southampton.
\end{abstract}

\section{INTRODUCTION}

Driver behavior has a significant effect on fuel consumption, a fact that was recognized as early as the 1970s [1]. In recent years, concerns about climate change have led to efforts to reduce anthropogenic greenhouse gas emissions, with several interventions being suggested as 'low-hanging fruit' that can produce a significant benefit at a low cost [2]. Economical driving behavior, or 'eco-driving', has been suggested as such a change, which could reduce fuel consumption and $\mathrm{CO}_{2}$ emissions from road vehicles by $10 \%$ with current technology. This is accomplished by encouraging drivers to accelerate moderately, anticipate signals and traffic flow to avoid stops, maintain an even speed, and to avoid idling [3].

Training programs have been devised to encourage ecodriving behaviors, with significant positive results [4]. However, the lowering of fuel consumption for commercial drivers in such training courses is often not reflected in their driving behavior after the end of the course [5]. In addition, some drivers revert back to their original driving styles in the months following the course [6], and conflicting goals such as reaching the destination in minimum time may reduce the effectiveness of encouraging drivers to save fuel [7]. Among untrained individuals, attitudes to eco-driving are generally positive but knowledge of specific fuel-saving behaviours is low [8].

A further complication for eco-driving is that avoiding sudden stops in traffic and at intersections is recognized as difficult for human drivers alone, as it requires prediction of future traffic flow and signal changes [1]. Reducing vehicle speed by coasting reduces fuel consumption, but this requires knowledge of the timing of signal changes and an accurate model of vehicle fuel consumption [9]. As such it is unrealistic that an unaided human driver could achieve minimum fuel usage. Similar coasting behaviors, termed 'pulse-and-glide' techniques, are known to reduce fuel consumption in vehiclefollowing situations, but exploitation of this has required automatic control systems [10]. On the other hand, techniques are known to calculate optimal speed profiles when the problem is formulated in an optimal control framework [11].

A potential remedy to the problem of reversion of drivers' behavioral changes after eco-driving training is to provide regular feedback to the driver on their behavior, for instance by using auditory, visual or haptic interfaces placed within a vehicle [12], [13]. These interfaces can be integrated with optimization of fuel consumption in order to find fuel-optimal behavior, which has been prototyped for the case that the vehicle travels on a dedicated route free of traffic [14]. Such eco-driving advanced driver assistance systems (ADAS) are seen as useful by European drivers and their deployment would likely be welcomed [15], but their user acceptance is dependent on the perceived disturbance to the driver [16].

To minimize disturbance and hence maximize user acceptance, an eco-driving assistance system could adapt to a particular user's driving style, a concept that has already been explored in the context of approaching intersections [17]. But to the best of the author's knowledge, no general eco-driving assistance system, or 'EDAS', has been developed to date that explicitly optimizes a model of driver preferences while also minimizing fuel consumption in real time.

In the current work, we introduce a framework in which the driver preferences and fuel consumption are both incorporated into the cost function of an optimal control problem. The modeling of driver preferences takes into account acceleration, velocity, spacing to other vehicles and cornering speed. Preferences are represented as penalty functions characterized by a small number of physically-meaningful parameters, and fuel consumption is modeled by fitting a bivariate polynomial to a known map. This optimal control problem is solved in realtime using a receding-horizon approach and the results used to update a visual interface on the speedometer of a vehicle to promote eco-driving behavior. Importantly, the use of optimal control as a mathematical framework allows for a trade-off 
to be made between driver preferences and fuel savings by altering a weighting factor in the optimal control problem. The authors recently developed a prototype of this system in a driving simulator, and we describe implementation details as well as the preliminary results of a pilot study carried out to evaluate the system.

\section{METHOD}

\section{A. Optimal control}

Optimal control is an extension of the calculus of variations that considers optimization of a performance criterion for a system by choosing its input. A good introduction to the subject can be found in [18], with more modern developments available in [19]. For the purposes of developing an ecodriving assistance system, it is a mathematical framework in which to formulate the problem of controlling vehicle acceleration to achieve minimum fuel usage. It also provides a straightforward way to achieve a trade-off between the objectives of minimizing fuel consumption and that of satisfying driver preferences. To accomplish this we consider minimizing a cost which is a weighted sum of a term $L_{f}$ penalizing fuel usage, and a term $L_{d}$ which represents driver preferences on speed and acceleration:

$$
J=\int_{T_{i}}^{T_{f}}\left(L_{d}+\alpha L_{f}\right) \mathrm{dt}
$$

If the term $L_{d}$ is chosen so that it is large when the driver is dissatisfied with the vehicle motion, then choosing a small value of $\alpha$ will prioritize driver preferences when (1) is minimized, while choosing a large value of $\alpha$ will prioritize fuel savings.

In receding horizon control, also known as model predictive control (MPC), this cost function is minimized considering a shorter time horizon $T$, and the optimization is repeated periodically, updating the system input as new values are calculated. A practical introduction to many MPC techniques may be found in [20]. We also consider the possibility of adding an additional term $\phi_{T}$ to the cost to partially compensate for the effect of shortening the horizon. Typically in MPC, this corresponds to the cost of some known, suboptimal control policy over the time interval $T$ to $T_{f}$. Denoting the time at which an optimization is carried out as $t=0$, this leads to the alternative cost function

$$
J_{\mathrm{MPC}}=\int_{0}^{T}\left(L_{d}+\alpha L_{f}\right) \mathrm{dt}+\phi_{T}
$$

which is to be minimized subject to the dynamics and current state of the vehicle. Considering this shortened horizon has the advantage that the optimization becomes more computationally tractable, as well as providing feedback to unmodeled dynamics and disturbances by repeating the optimization when new information is available.

\section{B. Vehicle dynamics}

The vehicle is considered as a mass that accelerates in response to four forces: the driving force provided by the engine, $f_{e}$, a negative retarding force given by the brakes, $f_{b}$, and air and rolling resistance. The driving force $f_{e}$ is nonnegative, and we may ensure this by adding inequality constraints to the optimal control formulation:

$$
f_{e} \geq 0
$$

Similarly, the braking force $f_{b}$ is nonpositive:

$$
f_{b} \leq 0
$$

By Newton's second law, the acceleration can be expressed as

$$
\dot{v}=\frac{1}{m}\left(f_{e}+f_{b}-c_{d} v^{2}-c_{r} m\right)
$$

where $v$ denotes vehicle velocity, $c_{d}=\frac{1}{2} \rho C_{d} A$ and $c_{r}=C_{r} g$ denote coefficients of drag and rolling resistance respectively, $\rho$ is the density of air, and $m$ and $A$ are the mass and frontal area of the vehicle respectively. The position of the vehicle is denoted by $x$ and varies in accordance with the velocity as:

$$
\dot{x}=v
$$

In addition to the position and velocity of the ego vehicle, the model of driver preferences includes a term depending on the distance to the vehicle in front of the driver, which we call the lead vehicle. As such we also consider the position of the lead vehicle as a state of the system that varies as

$$
\dot{x}_{l}=v_{l}
$$

where the leader velocity $v_{l}$ is assumed constant and is updated each time the optimization is solved. Including this term in our system dynamics can be interpreted as forming a prediction of the lead vehicle position under the assumption that it continues at its current speed.

\section{Fuel consumption model}

The fuel consumption model is based on a map of instantaneous fuel consumption generated by the $1 \mathrm{~d}$ combustion simulation software Ricardo Wave. The corresponding efficiency map is shown in Figure 1 and incorporates a constraint on maximum engine torque which depends on crankshaft speed. The map was modified to include the effect of clutch slip at crankshaft speeds of below 100 rad/s by noting that for a clutch in a manual transmission, we have an equilibrium of torques and hence all power loss occurs due to clutch slip. Therefore, if the clutch is allowed to slip at crankshaft speeds of below 100rad/s, the fuel consumption will be equal to that at $100 \mathrm{rad} / \mathrm{s}$ for a given torque. This causes the rapid deterioration in efficiency at low engine speeds visible in Figure 1.

Linear regression was used to approximate the fuel usage map by a polynomial, which is third order in engine torque $T_{e}$ and first order in crankshaft speed $\omega$ :

$$
\begin{aligned}
q_{f}(T, \omega)=p_{01} T+p_{10} \omega & +p_{11} T \omega \\
& +p_{02} T^{2}+p_{12} T^{2} \omega+p_{03} T^{3}
\end{aligned}
$$

This provides a smooth approximation to the true fuel map while requiring only 6 coefficients to be specified for implementation. Although a simulated fuel map was used in 


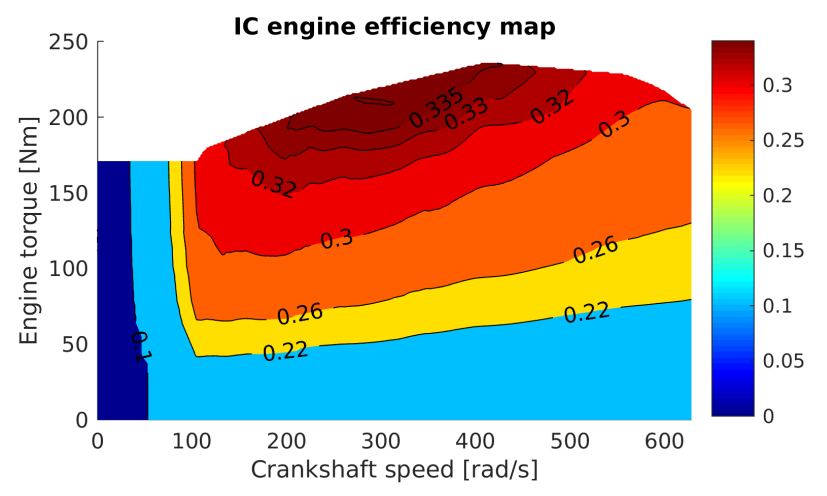

Fig. 1. Efficiency map for the engine model

\begin{tabular}{l|l} 
Coefficient & Value \\
\hline$p_{01}$ & $2.79 \times 10^{0}$ \\
$p_{10}$ & $2.59 \times 10^{-3}$ \\
$p_{11}$ & $6.81 \times 10^{-2}$ \\
$p_{02}$ & $-2.27 \times 10^{1}$ \\
$p_{12}$ & $-7.71 \times 10^{-2}$ \\
$p_{03}$ & $6.84 \times 10^{-1}$
\end{tabular}

TABLE I

COEFFICIENTS OF FUEL USAGE POLYNOMIAL

our implemetation, it would also be possible to fit the above function to real-world engine data. The coefficients used in our simulator implemetation are shown in Table I.

By considering the current gear ratio of the vehicle, $N$, and the rolling radius $r$ of the driven wheels, we can express the engine torque and crankshaft speed in terms of the driving force $f_{e}$ and vehicle velocity $v$ :

$$
T=\frac{r f_{e}}{N}, \quad \omega=\frac{N v}{r}
$$

This results in a stage cost for fuel consumption given by

$$
L_{f}=q_{f}\left(\frac{r f_{e}}{N}, \frac{N v}{r}\right)
$$

where $q_{f}$, the function for instantaneous fuel mass flow rate, is given by (3).

\section{Driver modeling}

We consider a model of driver preferences that considers penalty functions for harsh accelerations, for deviating from a desired velocity $v_{d}$, for maintaining a spacing to the lead vehicle that is too small or too large, and specifies a maximum speed when cornering that is dependent on road curvature. We denote the penalty functions for acceleration, velocity and spacing by $\psi_{a}, \psi_{v}$ and $\psi_{s}$ respectively.

The penalty function for acceleration is chosen to be quadratic, according to the formula,

$$
\psi_{a}(u)=u^{2}
$$

where we denote vehicle acceleration by $u$. In addition to this, a constraint is added to the model so that the vehicle acceleration is bounded

$$
b \leq u \leq a
$$

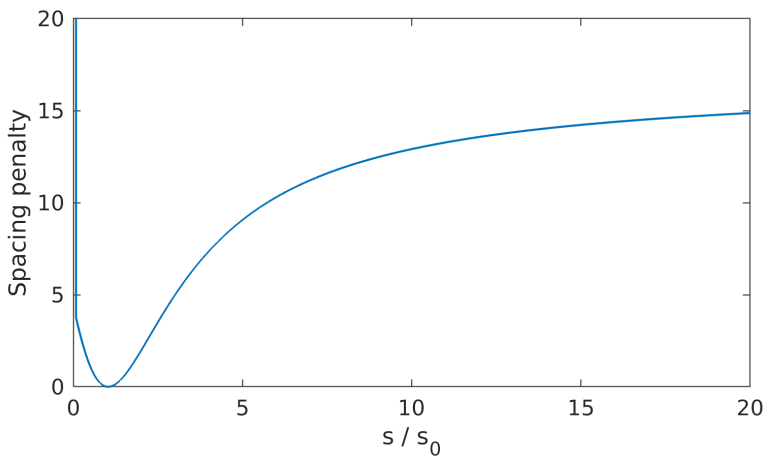

Fig. 2. Penalty function for vehicle spacing

where the constants $a$ and $b$ are parameters characterizing the driver. Similarly, we use a quadratic penalty for the velocity

$$
\psi_{v}(v)=\beta\left(v-v_{d}\right)^{2}
$$

where a weighting factor $\beta$ has been introduced. To choose the weighting factor, simulations were performed and the resulting vehicle accelerations compared with naturalistic driving data collected as part of the G-ACTIVE project [21]. The value $\beta=4 / v_{d}$ was found to give a good fit to real-world data and was used in the simulator implementation.

To describe driver preferences on inter-vehicle spacing $s=$ $x_{l}-x$, we introduce a penalty function that becomes large for small spacing values, but tends to a constant value as the spacing becomes large to represent the driver's indifference to far away vehicles. Considering the spacing relative to a desired spacing value $s_{d}$, a penalty that accomplishes this is shown in Figure 2 and is given by

$$
\psi_{s}\left(\frac{s}{s_{d}}\right)=\frac{\left(1-s / s_{d}\right)^{2}}{\left(s / s_{d}\right)^{2}+1}
$$

where the desired spacing is scaled according to the current speed according to $s_{d}=T_{h} v$ where $T_{h}$ is a driver parameter denoting the desired time headway to lead vehicles. We incorporate an additional constraint on the spacing

$$
s \geq 0
$$

which avoids collisions in the optimized trajectory.

The overall penalty function for the driver preferences is

$$
\psi(s, u, v)=u^{2}+\frac{4}{v_{d}}\left(v-v_{d}\right)^{2}+\frac{\left(1-s / s_{d}\right)^{2}}{\left(s / s_{d}\right)^{2}+1}
$$

but, for implementation, this must be rewritten in terms of the system states and inputs. Therefore we define:

$$
L_{d}=\psi\left(x_{l}-x, \frac{f_{e}}{m}+\frac{f_{b}}{m}-\frac{c_{d}}{m} v^{2}-c_{r}, v\right)
$$

Finally, we include a position-dependent speed limit in the model by incorporating an inequality constraint

$$
v \leq v_{\max }(x)
$$

where the function $v_{\max }$ incorporates both the legal speed limit for the road and also a maximum speed limit for cornering adapted from the lateral acceleration margin model of [22]. 


\section{E. The complete optimal control problem}

Considering $x, v, x_{l}$ as system states, and $f_{e}, f_{b}$ as system inputs, the complete problem can now be expressed as

$$
\begin{aligned}
\min _{f_{e}, f_{b}} \quad \int_{0}^{T}\left[L_{d}\left(x, v, x_{l}, f_{e}, f_{b}\right)+\alpha L_{f}\left(v, f_{e}\right)\right] \mathrm{dt}+\phi\left(x_{T}\right) \\
\text { s. t. } \quad \dot{v}=\frac{f_{e}}{m}+\frac{f_{b}}{m}-\frac{c_{d}}{m} v^{2}-c_{r} \\
\dot{x}=v, \quad \dot{x}_{l}=v_{l} \\
b \leq \frac{f_{e}}{m}+\frac{f_{b}}{m}-\frac{c_{d}}{m} v^{2}-c_{r} \leq a \\
v \leq v_{\max }(x), \quad s \geq 0 \\
f_{e} \geq 0,
\end{aligned}
$$

where (7a) and (7b) give the system dynamics while (7c), (7d), and (7e) denote mixed state-input, state, and input constraints respectively. This optimal control problem is solved at regular intervals subject to the initial conditions for $x, v, x_{l}$ and $v_{l}$ obtained from the simulator. The terminal cost is

$$
\phi\left(x_{T}\right)=\gamma\left(T v_{\mathrm{av}}+x_{0}-x_{T}\right)^{2}
$$

where $v_{\mathrm{av}}$ is a target average speed, and $x_{0}$ and $x_{T}$ denote the initial and final ego-position. This may be interpreted as a penalty for saving fuel by reducing speed, since this would increase the full cost (1) over the neglected part of the time horizon from $T$ to $T_{f}$. As an example, this target average speed could be taken to be either the current legal speed limit, or the velocity of the leader vehicle, whichever is lower.

\section{IMPLEMENTATION}

The authors recently implemented a prototype of the described system within the Southampton University Driving Simulator (SUDS), based at the University of Southampton in the UK. We now describe some notable aspects of this implementation.

\section{A. Driving simulator}

An image of the SUDS simulator is shown in Figure 3. In SUDS, a study participant sits inside the cabin of a 2015 Land Rover Discovery Sport, while viewing a simulated roadway environment on three projector screens mounted in front of the vehicle. A fourth projector screen is placed behind the vehicle, and small LCD screens are placed on each side-view mirror, in order to simulate the view in the rear-view and sideview mirrors. Engine sounds are simulated using the vehicle's internal audio system. SUDS also has a separate control room from which an operator can monitor the participant's progress.

The simulator uses the software package STISIM, which runs on a Windows 7 PC. The simulation software may be extended using the 'OpenModule' system, which allows for the definition of functions in an external shared library (a .dll) that are called during the simulation. This external dynamic link library (DLL) is programmed and compiled using Visual Basic, and resides on the same PC used for solving the optimal control problem. This capability was used to send data about the vehicle and roadway state to an optimizer application to solve the optimal control problem in real-time.

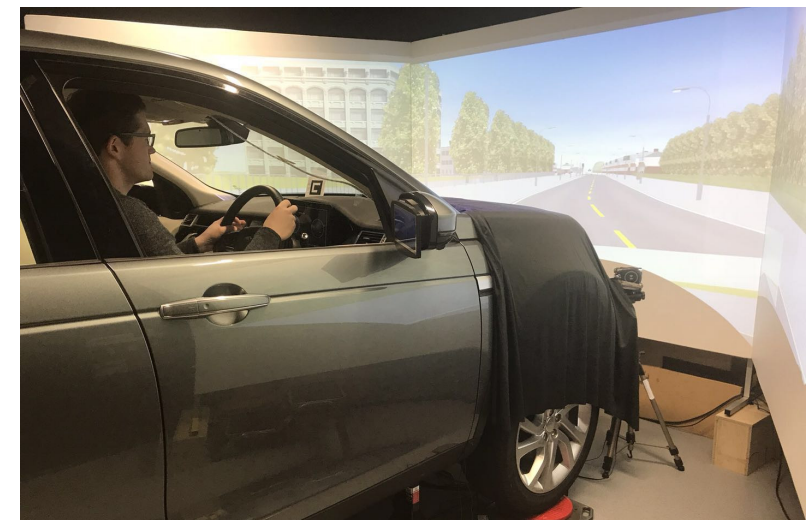

Fig. 3. The Southampton University Driving Simulator (SUDS)

\section{B. Receding-horizon control}

A receding-horizon control scheme was implemented by formulating the optimal control problem using the ACADO library [23]. This is incorporated into an optimizer application, which is written in $\mathrm{C}++$ and for this study was compiled using Visual Studio 2013. At regular intervals, this optimizer application receives the updated simulation state from STISIM/OpenModule, solves the optimal control problem, sends the updated velocity profile to the visual interface, and $\operatorname{logs}$ the results. For simplicity, we neglected the terminal cost (8) in the present implementation, as we found it to have little effect on the first part of the computed optimal trajectory if the prediction horizon length $T$ was chosen greater than approximately $20 \mathrm{~s}$.

The optimizer uses a BFGS approximation to the Hessian of the control problem and solves the problem at each time instant without any warm-starting, and in particular, does not use the code-generated real-time iteration MPC schemes that ACADO provides. This is due to incompatibility of the stage cost function with the least-squares formulation assumed by the ACADO nonlinear MPC code generation and results in longer computation times than could be expected from a well-designed nonlinear MPC implementation using realtime iterations. Nonetheless, we found that it is possible to solve the optimal control problem at a rate of $3-4 \mathrm{~Hz}$ for a 60 s time horizon and a $2 \mathrm{~s}$ discretization time-interval, which is sufficient for smooth updates to the recommended speed profile. This discretization is carried out using a fixed-step, 4th-order Runge-Kutta integrator.

\section{Visual interface design}

A visual interface was designed and developed in C\# using Windows Forms as a graphical library. The resulting application is executed on a Microsoft Surface Pro tablet, which is placed behind the steering wheel of the car to replace the physical instrument cluster. The visual design of the interface is shown in Figure 4, and comprises of a speed and RPM display augmented with a green and yellow 'eco-band' to recommend a near-optimal speed range to the driver. The green-region provides the user with a range of 


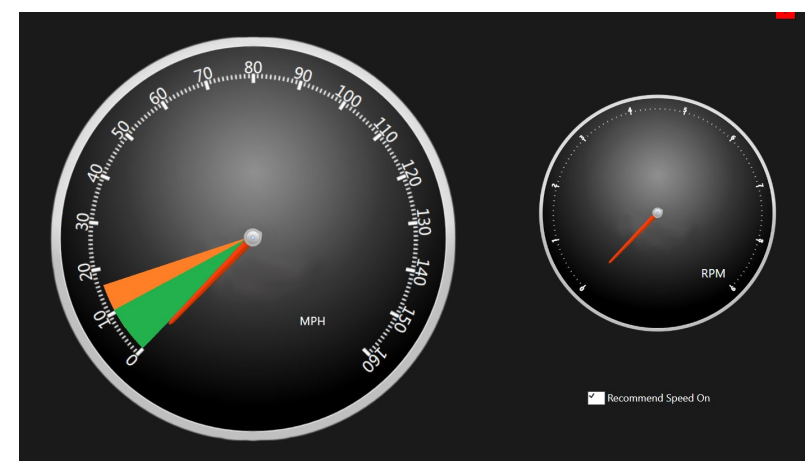

Fig. 4. The 'Dashboard' visual interface

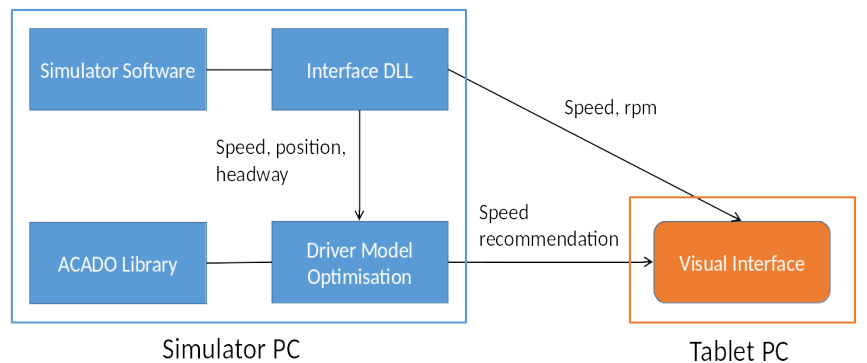

Fig. 5. Communication in the simulator setup

speeds recommended for fuel-efficient driving based on the current vehicle and road state. A further yellow region allows some margin for error and has a width chosen to correspond to typical speed variations observed in normal driving. A similar 'eco-speedometer' design was rated highly in perceived usefulness and user acceptance in a previous study [16].

When in use, the interface updates in real-time with the green eco-band stretching from zero speed to the current recommendation of the optimizer, which is taken as the egovehicle speed at $10 \mathrm{~s}$ into the horizon of the optimal control problem. As data is received from the optimizer application, this speed changes, which has the effect of smoothly interpolating between recommended speed values in order to gently 'coach' the driver into following the optimal speed profile.

\section{Networking and communication}

Communication between the various applications is carried out by sending TCP messages on the local WiFi network within SUDS. TCP was selected over UDP as a transmission protocol due to its error-checking capabilities and ability to recover from packet loss. This allows the OpenModule DLL to send the current simulator state, including vehicle positions and velocities, to the dashboard and the optimizer, and for the optimizer to send recommended velocities to the dashboard. Figure 5 shows a diagram of the data transfer between the different parts of the system.

The system has a modular design, and although the OpenModule DLL resides on the same PC as the optimizer for this simulator prototype, this would not be the case in a real-world implementation where sensor data and a state estimator would take the place of the simulator state data and OpenModule DLL. This modularity enables the different parts of the system to be reused and updated independently from one another. To migrate to different simulation software or real-world testing, only the DLL would have to be changed.

\section{EVALUATION}

Figure 6 shows examples of optimal speed profiles for the situation of accelerating from stationary and braking to a halt for different values of the weighting parameter $\alpha$. The fuel usage per kilometer for these speed profiles is shown in Table II. It is apparent that even small differences in speed profile can make significant improvements to fuel economy if coasting behavior is encouraged. Similar potential fuel economy improvements are found in other situations, with the largest potential for improvement occuring when coasting down before corners and intersections, especially if it is possible to avoid stopping by doing so.

For initial evaluation of the system, a pilot study was carried out in the simulator with 5 participants, in which each participant drove the same route in three different cases:

- A 'control' run in which the participant drove as they normally would,

- An 'eco-drive' run in which the participant was instructed to drive conserving fuel,

- An 'eco-band' run in which the participant drove with the visual interface turned on,

This pilot study was designed to gather user feedback and identify any problems with the system setup. We did not attempt to draw any conclusions about the effectiveness of the system from this pilot study as the sample size is too small to do so, but this will be evaluated in future work by conducting a larger-scale study involving 36 participants.

Study participants were asked, after each test run, to fill in several questionnaires relating to system usability and cognitive workload. In addition, several participants made comments which led to improvements to the interface and simulator setup, including:

- That the motion of the band in the interface was not smooth and sometimes 'jumped' from one value to another, especially before corners.

- That the simulated traffic on the test route sometimes behaved in unexpected ways, such as by remaining stationary at an intersection rather than moving off.

These issues were addressed in work carried out after the study, which included altering the traffic on the simulated route, making some computational efficiency improvements to the optimizer to allow for a longer prediction horizon (60s instead of 30s), and interpolating the eco-band position to provide smoother motion.

\section{COnClusion And Future WORK}

The present work presents a possible optimal control framework for ADAS designed to promote eco-driving and fuelefficiency. This framework considers both a simplified model 

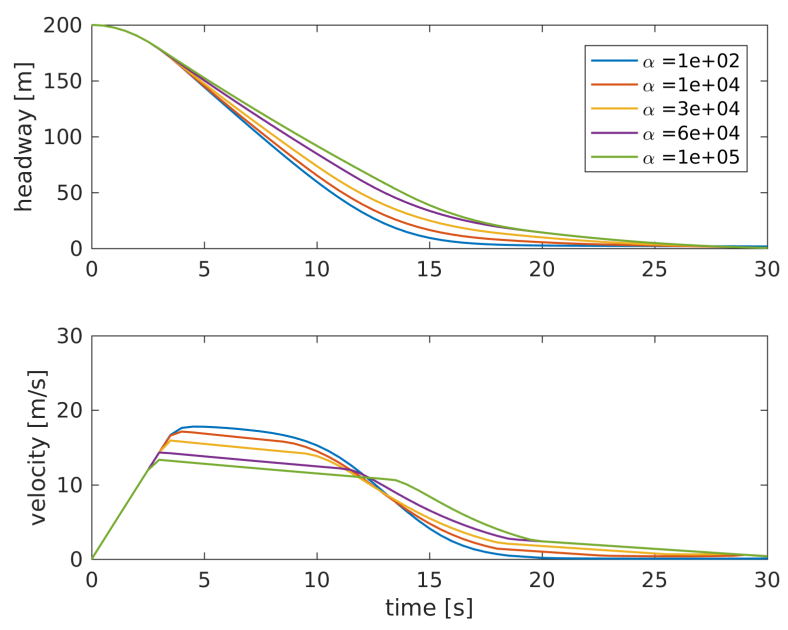

Fig. 6. Example optimized speed profiles

\begin{tabular}{l|lllll}
$\alpha$ & $1 \mathrm{e} 2$ & $1 \mathrm{e} 4$ & $3 \mathrm{e} 4$ & $6 \mathrm{e} 4$ & $1 \mathrm{e} 5$ \\
\hline Fuel use g/km & 14.45 & 13.67 & 13.60 & 13.56 & 13.56 \\
Improvement & $0 \%$ & $5.69 \%$ & $6.26 \%$ & $6.54 \%$ & $6.57 \%$ \\
& \multicolumn{5}{c}{ TABLE II }
\end{tabular}

FUEL USAGE OF SPEED PROFILES

of the vehicle powertrain incorporating an engine map and a model of driver behavior in order to effectively trade-off fuel saving and driver preferences. Numerical simulations show that even small changes in driving speed profiles can produce large fuel savings if coasting behaviors are encouraged.

A prototype implementation of such an ADAS was made in a driving simulator, and a pilot study carried out to assess system performance. A key conclusion is that the real-time optimization of the driver's speed profile at a rate of $3-4 \mathrm{~Hz}$ is technically feasible. User feedback was used to improve the implementation, with a focus on comments about the smoothness of motion of the eco-band in the visual interface.

A larger-scale study will be carried out in future work to assess the effectiveness of the system in promoting fuelefficient, eco-driving behaviors. Future work will also consider other ways to present information about coasting to the driver. A key issue is to discover an effective method to prompt the driver when to start coasting down before intersections for improved fuel economy, which likely requires further development of the visual interface.

\section{ACKNOWLEDGMENT}

We gratefully acknowledge the support of the UK Engineering and Physical Sciences Research Council (EPSRC), under grant number EP/N022262/1: "Green Adaptive Control for Future Interconnected Vehicles".

\section{REFERENCES}

[1] L. Evans, "Driver behavior effects on fuel consumption in urban driving," in Proceedings of the Human Factors Society Annual Meeting, vol. 22, no. 1. Sage Publications Sage CA: Los Angeles, CA, 1978, pp. 437-442.
[2] M. P. Vandenbergh, J. Barkenbus, and J. Gilligan, "Individual carbon emissions: The low-hanging fruit," UCLA L. Rev., vol. 55, p. 1701, 2007.

[3] J. N. Barkenbus, "Eco-driving: An overlooked climate change initiative," Energy Policy, vol. 38, no. 2, pp. 762-769, 2010.

[4] M. Zarkadoula, G. Zoidis, and E. Tritopoulou, "Training urban bus drivers to promote smart driving: A note on a greek eco-driving pilot program," Transportation Research Part D: Transport and Environment, vol. 12 , no. 6, pp. 449-451, 2007.

[5] A. E. af Wåhlberg, "Long-term effects of training in economical driving: Fuel consumption, accidents, driver acceleration behavior and technical feedback," International Journal of Industrial Ergonomics, vol. 37, no. 4, pp. 333-343, 2007.

[6] B. Beusen, S. Broekx, T. Denys, C. Beckx, B. Degraeuwe, M. Gijsbers, K. Scheepers, L. Govaerts, R. Torfs, and L. I. Panis, "Using on-board logging devices to study the longer-term impact of an eco-driving course," Transportation research part D: transport and environment, vol. 14, no. 7, pp. 514-520, 2009.

[7] E. Dogan, L. Steg, and P. Delhomme, "The influence of multiple goals on driving behavior: The case of safety, time saving, and fuel saving," Accident Analysis \& Prevention, vol. 43, no. 5, pp. 1635-1643, 2011.

[8] R. C. Mcllroy and N. A. Stanton, "What do people know about ecodriving?" Ergonomics, vol. 60, no. 6, pp. 754-769, 2017.

[9] H. Rakha and R. K. Kamalanathsharma, "Eco-driving at signalized intersections using V2I communication," in Intelligent Transportation Systems (ITSC), 2011 14th International IEEE Conference on. IEEE, 2011, pp. 341-346.

[10] S. E. Li, H. Peng, K. Li, and J. Wang, "Minimum fuel control strategy in automated car-following scenarios," IEEE Trans. on Vehicular Technology, vol. 61, no. 3, pp. 998-1007, 2012.

[11] A. Sciarretta, G. De Nunzio, and L. L. Ojeda, "Optimal ecodriving control: Energy-efficient driving of road vehicles as an optimal control problem," IEEE Control Systems, vol. 35, no. 5, pp. 71-90, 2015.

[12] S. Azzi, G. Reymond, F. Mérienne, and A. Kemeny, "Eco-driving performance assessment with in-car visual and haptic feedback assistance," Journal of Computing and Information Science in Engineering, vol. 11, no. 4, p. 041005, 2011.

[13] R. C. McIlroy, N. A. Stanton, L. Godwin, and A. P. Wood, "Encouraging eco-driving with visual, auditory, and vibrotactile stimuli," IEEE Trans. on Human-Machine Systems, vol. 47, no. 5, pp. 661-672, 2017.

[14] Q. Cheng, L. Nouveliere, and O. Orfila, "A new eco-driving assistance system for a light vehicle: Energy management and speed optimization," in Intelligent Vehicles Symposium. IEEE, 2013, pp. 1434-1439.

[15] S. Trommer and A. Höltl, "Perceived usefulness of eco-driving assistance systems in Europe," IET Intelligent Transport Systems, vol. 6, no. 2 , pp. $145-152,2012$.

[16] A. Meschtscherjakov, D. Wilfinger, T. Scherndl, and M. Tscheligi, "Acceptance of future persuasive in-car interfaces towards a more economic driving behaviour," in Proceedings of the 1st International Conference on Automotive User Interfaces and Interactive Vehicular Applications. ACM, 2009, pp. 81-88.

[17] X. Xiang, K. Zhou, W.-B. Zhang, W. Qin, and Q. Mao, "A closedloop speed advisory model with driver's behavior adaptability for ecodriving," IEEE Trans. on Intelligent Transportation Systems, vol. 16, no. 6, pp. 3313-3324, 2015.

[18] A. E. Bryson and Y.-C. Ho, Applied optimal control: optimization, estimation and control. CRC Press, 1975.

[19] D. P. Bertsekas, Dynamic programming and optimal control. Athena scientific Belmont, MA, 1995, vol. 1, no. 2.

[20] E. F. Camacho and C. B. Alba, Model predictive control. Springer Science \& Business Media, 2013.

[21] X. Yan, J. Fleming, C. Allison, and R. Lot, "Portable Automobile Data Acquisition Module (ADAM) for naturalistic driving study," in 15th European Automotive Congress (EAEC 2017).

[22] G. Reymond, A. Kemeny, J. Droulez, and A. Berthoz, "Role of lateral acceleration in curve driving: Driver model and experiments on a real vehicle and a driving simulator," Human factors, vol. 43, no. 3, pp. 483-495, 2001.

[23] B. Houska, H. Ferreau, and M. Diehl, "ACADO Toolkit - An Open Source Framework for Automatic Control and Dynamic Optimization," Optimal Control Applications and Methods, vol. 32, no. 3, pp. 298-312, 2011. 